\title{
Variación temporal de la abundancia y algunos aspectos poblacionales de Micrathena (Araneae: Araneidae) en un Bosque Seco Tropical (BST) del Caribe colombiano
}

\author{
Luis Quijano-Cuervo ${ }^{12,1}$; Neis Martínez-Hernández ${ }^{1,2}$ \& Alexander Sabogal- \\ GONZÁLEZ
}

\begin{abstract}
${ }^{1}$ Semillero de investigación Artrópodos del Caribe Colombiano "NEOPTERA". Programa de Biología, Facultad de Ciencias Básicas, Universidad del Atlántico. Barranquilla, Colombia. ${ }^{2}$ Grupo Biodiversidad del Caribe colombiano. Programa de Biología, Facultad de Ciencias Básicas, Universidad del Atlántico. Barranquilla, Colombia. ${ }^{3}$ Centro de Investigaciones en Acarología, Bogotá, Colombia.
\end{abstract}

\begin{abstract}
Resumen. Se analizó la variación temporal de la abundancia y algunos aspectos poblacionales de especies simpátricas de arañas del género Micrathena en un fragmento de BST al norte de Colombia, entre noviembre de 2012 y agosto de 2013. Se delimitó una transecta lineal de $500 \mathrm{~m}$ de largo por $20 \mathrm{~m}$ de ancho, donde se instalaron 10 puntos distanciados $50 \mathrm{~m}$ entre sí. En cada punto, las arañas fueron capturadas empleando la captura manual y la agitación del follaje. Se capturaron 177 individuos, distribuidos en cuatro especies. $M$. schreibersi fue la más abundante (141 individuos) y M. horrida, la menos común. Se observó un patrón de variación temporal en tres de las cuatro especies del género estudiadas, caracterizado por la disminución de los individuos adultos durante la época seca y su aumento durante las lluvias. Adicionalmente, se encontró una diferencia marcada en las proporciones sexuales de cada especie, así como en el tamaño entre especies. Esto último podría sugerir, en parte, un patrón de segregación de nicho como mecanismo para evitar la competencia. Dada la relación compleja entre las especies de Micrathena y los factores ambientales evaluados, su fácil identificación, visualización y captura en campo, estas arañas se convierten en potenciales bioindicadoras del estado de conservación del BST. Por esta razón se recomienda su uso en futuros estudios de impacto ambiental.
\end{abstract}

[Palabras clave: arañas, neotrópico, precipitación, simpatría]

\begin{abstract}
Aвstract. Temporal variation of the abundance and some population aspects of Micrathena species (Araneae: Araneidae) in a Dry Tropical Forest (DTF) from Colombian Caribbean. The temporal variation in abundance and some population aspects of sympatric spider species of genus Micrathena in a dry tropical forest fragment in northern Colombia between November 2012 and August 2013 were analyzed. A linear transects of $500 \mathrm{~m}$ length and $20 \mathrm{~m}$ wide were delimitated, where 10 points were installed spaced $50 \mathrm{~m}$. In each point, spiders were captured using manual capture and foliage agitation. 177 individuals were captured, distributed into four species, where $M$. schreibersi was the most abundant with 141 individuals and M. horrida the least common. A pattern of temporal variation was observed in three of the four species studied; characterized by decline of adult individuals during the dry season and increase during the rainy months. Additionally, a marked difference in sex ratios of each species, as well as the size between species, were found; the latter may in part suggest a pattern of niche segregation as a mechanism to avoid competition. Given the complex relationship of the Micrathena species with the environmental factors evaluated, their easy identification, visualization and capture in the field, these spiders become potential bioindicators of the state of conservation of DTF, so its use is recommended in future studies of environmental impact.
\end{abstract}

[Keywords: spiders, neotropic, precipitation, sympatry]

\section{INTRODUCCIÓN}

La variación temporal en la abundancia de los artrópodos es un fenómeno común en los ecosistemas naturales, y generalmente se la asocia a la variabilidad climática de la región que habitan (Wolda 1978; Pinheiro et al. 2002). Dicha variación puede explicarse por la interacción entre factores abióticos y bióticos tales como la disponibilidad de presas, la fenología del bosque, la depredación, la temperatura, la humedad y

Editor asociado: Alejandro Farji-Brener

luisquijanocuervo@gmail.com la precipitación (Didham and Springate 2003; Grimbacher and Stork 2009; Vasconcellos et al. 2010; Beltrán and Wunderle 2014). Estos dos últimos factores son los que más influencian los patrones temporales (Wolda 1988). Dentro de los artrópodos sobre los que se ha analizado la dinámica temporal de su abundancia o la diversidad, encontramos a las arañas. Éstas demostraron ser sensibles a los cambios ambientales, y exhiben patrones temporales marcados (Lubin 1978; Giraldo et al. 2004; Sudhikumar et al. 2005; Mineo et al. 
2010; Beltrán and Wunderle 2014; Campuzano et al. 2016).

Dentro de las arañas se encuentra el género Micrathena (Sundevall, 1833). Este es un grupo común de arañas diurnas, endémicas de América (Levi 1985), que se caracterizan por su fácil identificación, visualización y captura en campo. Estos las convierte en un grupo ideal para estudios ecológicos (Sabogal and Flórez 2000). En Colombia se han registrado 58 (Barriga and Moreno 2013) de las 119 especies descriptas dentro de este género (WSC 2017), distribuidas en una amplia diversidad de hábitats entre 0 y $3000 \mathrm{~m}$ s. n. m. (Sabogal and Flórez 2000). En algunas investigaciones se ha detectado la coexistencia temporal y espacial de varias especies de este género, además de la dinámica estacional de sus abundancias, relacionada con el régimen de lluvias (Blanco et al. 2003; Ferreira-Ojeda et al. 2009; Escorcia et al. 2012). La mayoría de estudios que evalúan la dinámica poblacional de Micrathena se han desarrollado con $M$. gracilis (Walckenaer, 1805) en Norteamérica (Uetz and Hartsock 1986; Hodge 1987a,b; Bukowski and Christenson 1997; Bukowski and Christenson 2000; Vanderhoff et al. 2008; McCravy and Hessler 2012), mientras que en el Neotrópico sólo figuran los desarrollados con M. schreibersi (Perty, 1833) en Panamá (Shelly, 1984), M. sexspinosa (Hahn, 1822) en Costa Rica (Eberhard, 1988) y Panamá (Gálvez, 2011), y M. funebris (Marx, 1898) en México (Melling-López et al. 2008). Esto indica que se desconocen los aspectos poblacionales de la mayoría de especies del género.

En general, las especies de este género han sido registradas tanto en bosques húmedos (Lubin 1978; Shelly 1984; Eberhard 1988; Ferreira-Ojeda et al. 2009; Gálvez 2011; McHugh et al. 2014) como en bosques secos (Meling-López et al. 2008; Escorcia et al. 2012). En los bosques secos, la información sobre la ecología de artrópodos (en particular arañas) es escasa, a pesar de ser un ecosistema con alto grado de vulnerabilidad y fragmentación. Por esta razón, el presente trabajo analiza la dinámica temporal de las abundancias y algunos aspectos poblacionales de cuatro especies de Micrathena presentes en un fragmento de bosque seco tropical del Caribe Colombiano.

\section{Materiales y Métodos}

\section{Área de estudio}

La Reserva Campesina La Montaña (RCM) está ubicada entre los municipios de Juan de
Acosta y Usiacurí, departamento del Atlántico, Colombia (Figuras 1 y 2). Está localizada en $10^{\circ} 46^{\prime} 02^{\prime} 06^{\prime \prime} \mathrm{N}$ y $75^{\circ} 02^{\prime} 34^{\prime \prime} \mathrm{O}$, a una altura que oscila entre 160 y $250 \mathrm{~m}$. La zona boscosa cuenta con una extensión aproximada de 47 ha y fue declarada reserva campesina en 1996 según el Decreto 1777. Esta área de bosque integra una formación higrotropofítica correspondiente a un bosque seco tropical (BST) dominado por especies de plantas como Hura crepitans L. y Terminalia amazonia L. (Berdugo-Latke et al. 2012). Según la clasificación climática propuesta por Rangel-CH and CarvajalCogollo (2012) para la región Caribe, la RCM se encuentra dentro de la unidad climática B, subunidad climática B1 (distribución delluvias unimodal-biestacional), con una precipitación anual total de $1052 \mathrm{~mm}$ y una media mensual de $88 \mathrm{~mm}$. La concentración de las lluvias en esta subunidad se presenta entre mayo y noviembre. Octubre es el mes más lluvioso, con $170 \mathrm{~mm}$. La temperatura promedio del lugar es $27.6^{\circ} \mathrm{C}$ y la humedad relativa promedio es $62 \%$, con pocas variaciones a lo largo del año (García-Atencia and MartínezHernández 2015).

\section{Diseño de muestreo}

Se realizaron 10 muestreos entre noviembre de 2012 y agosto de 2013, con salidas mensuales de $48 \mathrm{~h}$. Los muestreos fueron realizados entre las 8:00 y 12:00 y las 13:30 y 18:00 h, completando un esfuerzo de 18 horas/hombre. En el interior del fragmento de la RCM, cubriendo zonas de interior de bosque y vegetación riparia aledaña a una quebrada temporal, se delimitó una transecta lineal de $500 \mathrm{~m}$ de largo por $20 \mathrm{~m}$ de ancho, donde se marcaron 10 puntos de muestreo distanciados $50 \mathrm{~m}$ entre sí. Para capturar las arañas espinosas del género Micrathena, en cada punto se utilizaron las técnicas de captura manual (CM) y agitación del follaje (AF). La primera técnica consistió en la búsqueda de los individuos durante un tiempo aproximado de 30 minutos en un radio de acción de 10 m por punto, para un total de 300 minutos por muestreo. Por otro lado, la AF se efectuó con un esfuerzo muestral de 60 agitaciones por punto, en todos los estratos posibles de inventariar, excluyendo el dosel. Para esto se utilizó una red entomológica de $60 \mathrm{~cm}$ de diámetro por $20 \mathrm{~cm}$ de profundidad y un madero para sacudir la vegetación.

Los individuos recolectados fueron preservados en alcohol etílico al $70 \%$ e identificados con ayuda de las claves de 


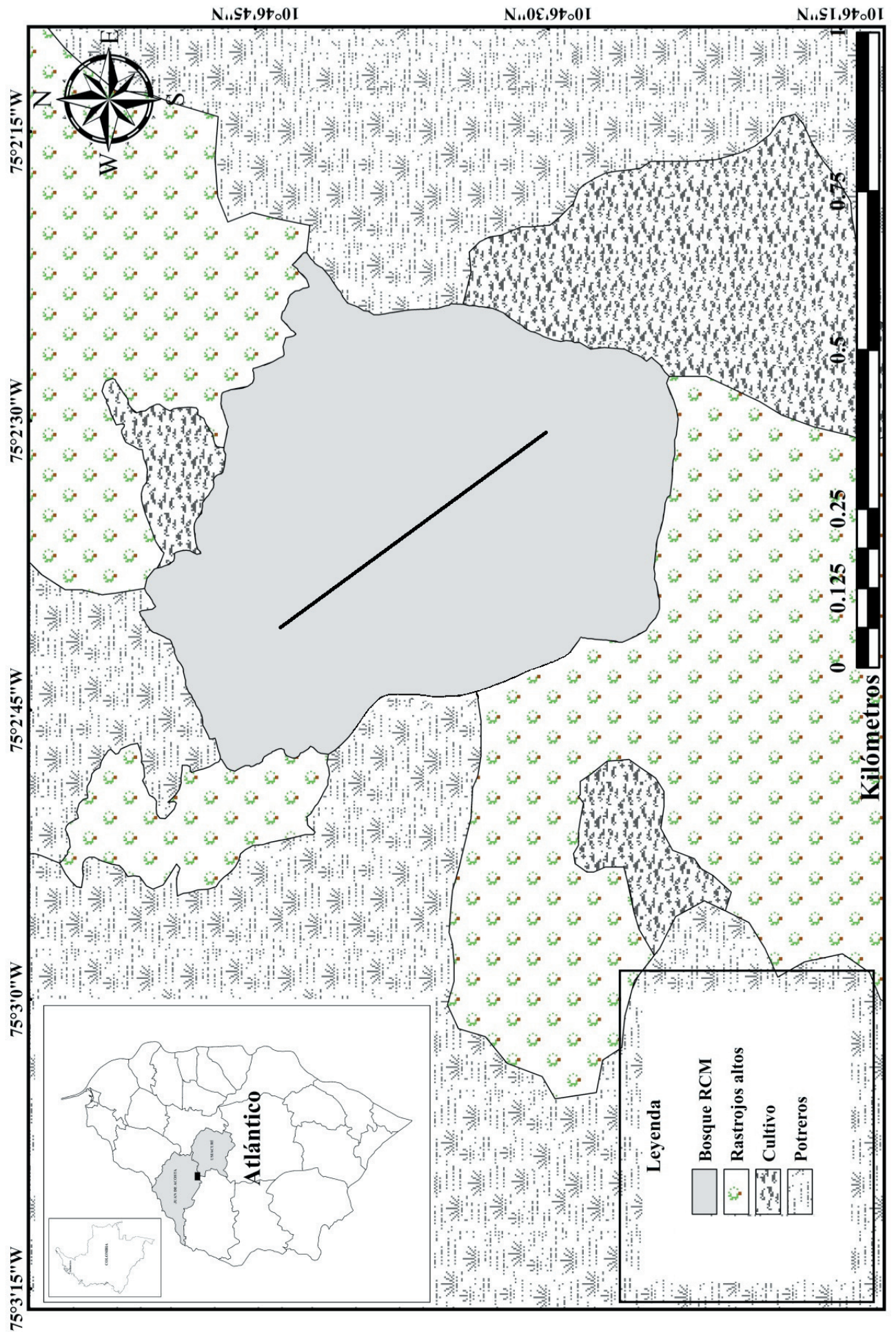

Figura 1. Localización geográfica de la Reserva Campesina La Montaña (RCM), Juan de Acosta, Atlántico, Colombia. Tomado y modificado de: Rangel-Acosta et al. 2016.

Figure 1. Geographical location of the Reserva Campesina La Montaña (RCM), Juan de Acosta, Atlántico, Colombia. Modify from Rangel-Acosta et al. 2016. 
Levi $(1985,2002)$. Posteriormente fueron confirmados con la colección de arañas del Instituto de Ciencias Naturales (ICN) de la Universidad Nacional-Sede Bogotá y se los incorporó al museo de este instituto y a la colección de arañas en húmedo del Museo de la Universidad del Atlántico (UARC). A todos los individuos de cada especie se les midió el largo (LO) y ancho (AO) del opistosoma con un calibrador digital. Estas medidas fueron seleccionadas con base en Meling-López et al. (2008) y Magalhães and Santos (2011).

Por último, en cada uno de los puntos durante todos los meses de muestreo se midieron variables ambientales "in situ" como la humedad relativa y la temperatura ambiental con un termohigrómetro (marca Extech), y la precipitación con un pluviómetro (marca Hellmam) instalado en los alrededores de la reserva. De esta última variable se obtuvieron valores totales mensuales correspondientes a la suma de cada uno de los eventos de precipitación ocurridos durante cada mes.

\section{Análisis de Datos}

Se estimó la abundancia como el número de individuos por sexo de cada una de las especies. Con base en la abundancia relativa de los sexos, se calcularon valores de proporción. Por último, para establecer posibles relaciones entre las variables ambientales y las abundancias relativas de las especies de Micrathena, se realizó un análisis de correlación de Spearman, esto con la ayuda del Programa Past 3.10 (Hammer et al. 2001).
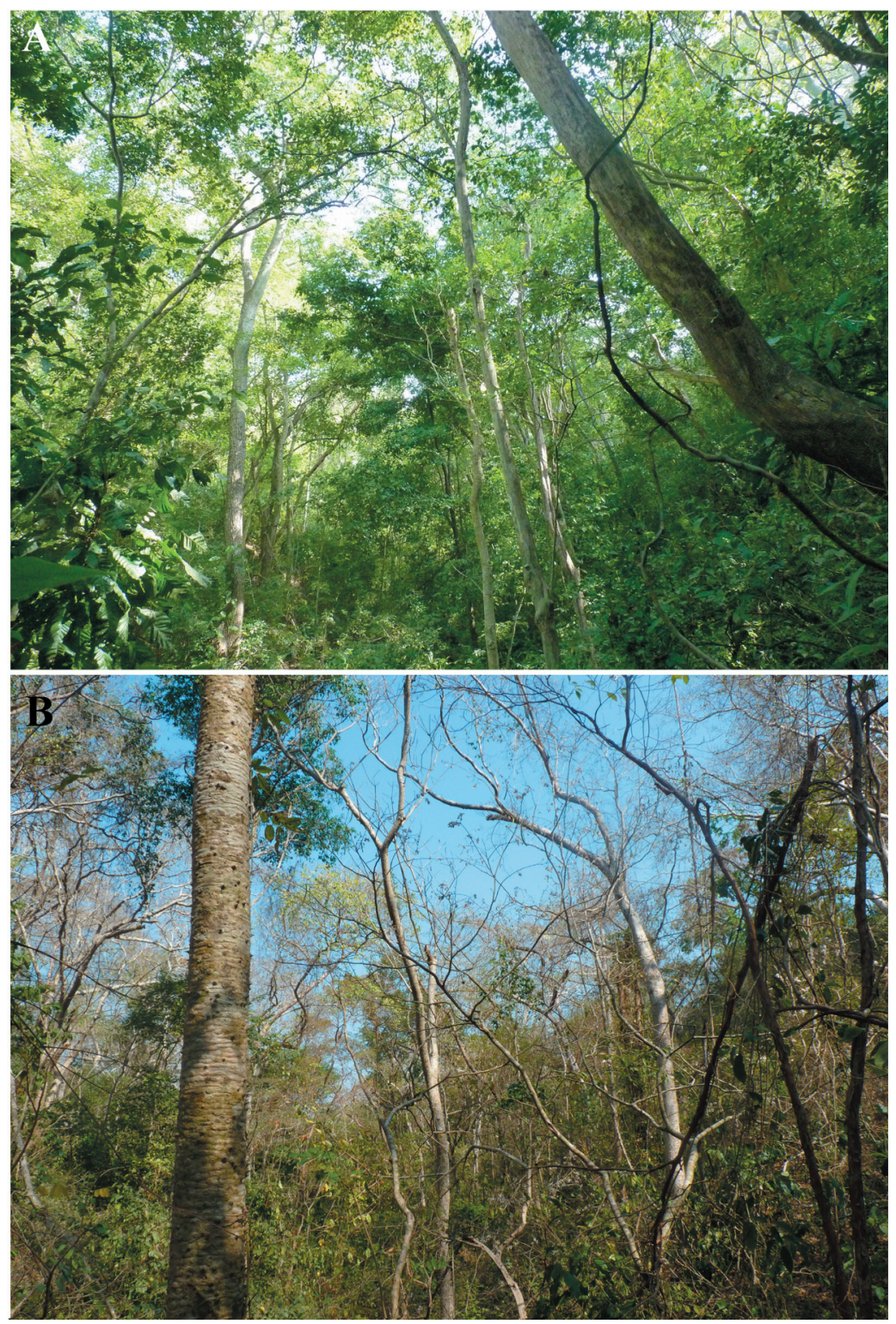

Figura 2. Vista de la RCM durante las temporadas de lluvia (A) y seca (B).

Figure 2. View of the RCM during the rainy (A) and dry 


\section{Resultados}

Se capturaron 177 individuos distribuidos en cuatro especies, entre las que M. schreibersi (Perty 1833) se destacó como la más abundante (141 individuos, 79.7\%) (Figura 3A y B), seguida por M. sexspinosa (Hahn 1822) (24 individuos, 13.5\%) (Figura 3C y D) y por $M$. quadriserrata F.O. Pickard-Cambridge, 1904 (11 individuos, 6.2\%) (Figura 3E y F). Con estos registros se confirma la presencia de esta última especie en Colombia, que había sido sugerida por Sabogal and Flórez (2000). Por último, la especie menos abundante fue $M$. horrida (Taczanowski, 1873) con un individuo hembra capturado en abril (Tabla 1, Figura 3G y H). La mayor abundancia de $M$. schreibersi (37 individuos) se registró en mayo; $M$. sexspinosa fue más abundante en diciembre (16 individuos) y $M$. quadriserrata lo fue en mayo y agosto (3 individuos). Por otro lado, del total de la abundancia reportada para las cuatro especies, $53.1 \%$ correspondió a individuos inmaduros (juveniles y subadultos), entre los que M. schreibersi presentó la mayor cantidad (84 individuos). En cuanto a la proporción

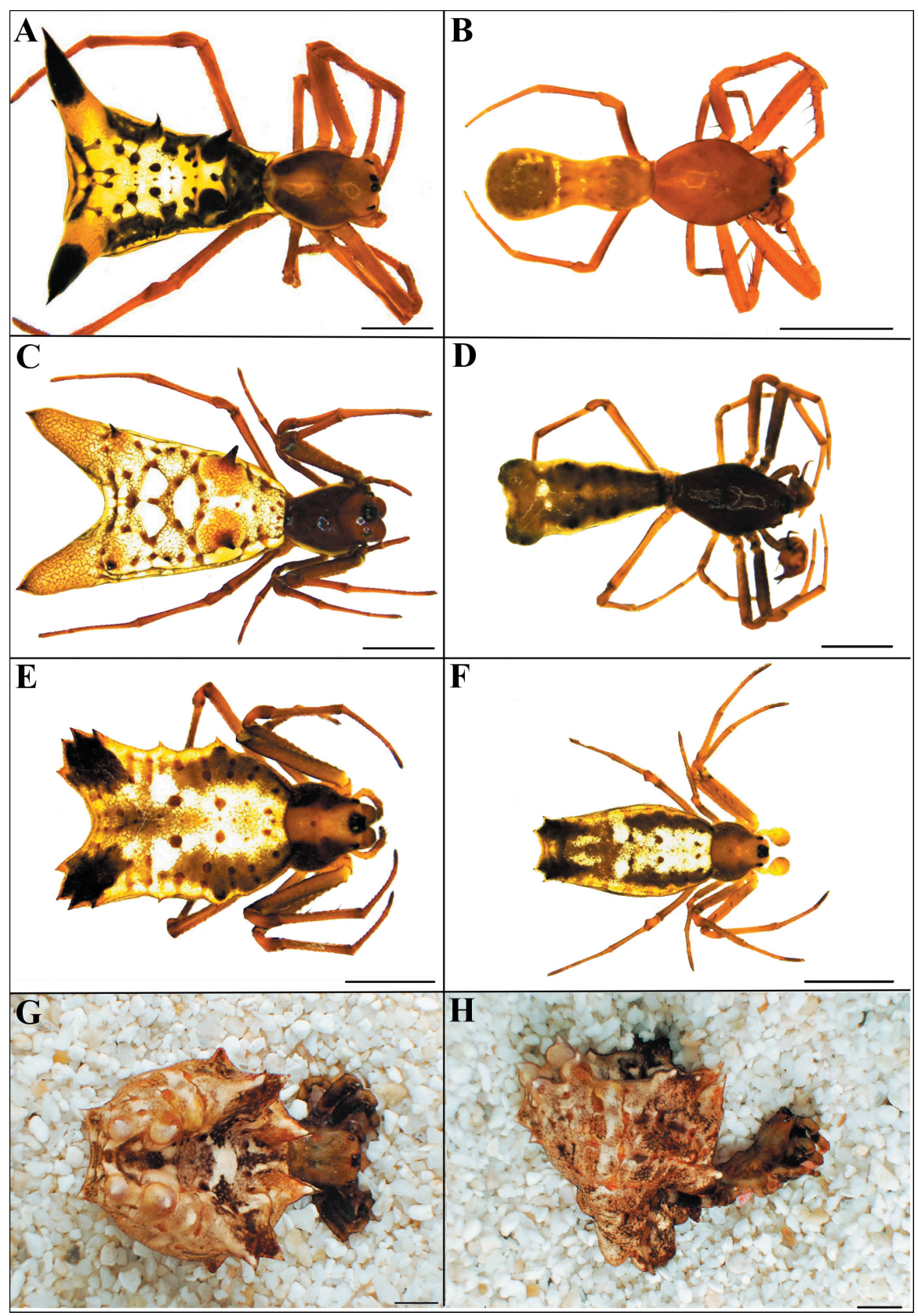

Figura 3. Especies de Micrathena capturadas en la RCM. A: $M$. schreibersi-hembra, B: M. schreibersi-macho, C: M. sexspinosahembra, D: $M$. sexspinosa-macho, E: $M$. quadriserratahembra, F: $M$. quadriserrata-macho, G: $M$. horrida-hembra vista dorsal, H: G: $M$. horrida-hembra vista lateral.

Figure 3. Micrathena species captured in the RCM. A: $M$. schreibersi-female, B: M. schreibersi-male, C: M. sexspinosafemale D: $M$. sexspinosa-male E: $M$. quadriserrata-female F: M. quadriserrata-male G: $M$. horrida-female dorsal view, H: G: $M$. horrida-female lateral view. 
Tabla 1. Abundancia mensual de Micrathena y variables ambientales en la RCM. Abreviaturas. Nov: noviembre; Dic: diciembre; Ene: enero; Feb: febrero; Mar: marzo; Abr: abril; May: mayo; Jun: junio; Jul: julio; Ago: agosto; Prec (mm): precipitación; H.R (\%): humedad relativa; T.A $\left({ }^{\circ} \mathrm{C}\right)$ : temperatura ambiente.

Table 1. Monthly abundance of Micrathena and environmental variables in the RCM. Abbreviations. Nov: November; Dic: December; Ene: January; Feb: February; Mar: March; Abr: April; May: May; Jun: June; Jul: July; Ago: August; Prec $(\mathrm{mm})$ : precipitation; H.R. (\%): relative humidity; T.A. $\left({ }^{\circ} \mathrm{C}\right)$ : ambient temperature.

\begin{tabular}{|c|c|c|c|c|c|c|c|c|c|c|c|}
\hline \multirow[t]{2}{*}{ Especies-Variables } & \multicolumn{10}{|c|}{ Meses } & \multirow[t]{2}{*}{ Total } \\
\hline & Nov & Dic & Ene & Feb & Mar & Abr & May & Jun & Jul & Ago & \\
\hline M. schreibersi & 14 & 19 & 3 & 2 & 14 & 24 & 37 & 11 & 10 & 7 & 141 \\
\hline M. sexspinosa & 2 & 16 & 0 & 0 & 0 & 0 & 1 & 4 & 0 & 1 & 24 \\
\hline M. quadriserrata & 0 & 2 & 0 & 0 & 0 & 1 & 3 & 2 & 0 & 3 & 11 \\
\hline M. horrida & 0 & 0 & 0 & 0 & 0 & 1 & 0 & 0 & 0 & 0 & 1 \\
\hline Riqueza & 2 & 3 & 1 & 1 & 1 & 3 & 3 & 3 & 1 & 3 & 4 \\
\hline Abundancia total & 16 & 37 & 3 & 2 & 14 & 26 & 41 & 17 & 10 & 11 & 177 \\
\hline Prec. (mm) & 351 & 33 & 0 & 0 & 8 & 63 & 78 & 99 & 21 & 6 & \\
\hline H.R. (\%) & 79.2 & 75.4 & 60.8 & 52.6 & 56.5 & 82.2 & 83.4 & 80 & 76.9 & 84.5 & \\
\hline T.A. $\left({ }^{\circ} \mathrm{C}\right)$ & 29.3 & 30.7 & 31.9 & 32.6 & 35.5 & 25.9 & 26.3 & 33.3 & 30.6 & 29.2 & \\
\hline
\end{tabular}

sexual, se observó una dominancia clara de las hembras en $M$. sexspinosa (9:1) y M. schreibersi (3:1), mientras que $M$. quadriserrata estuvo más cercana al valor teórico $(2: 1)$.

En relación a la variación temporal de las poblaciones estudiadas, de manera general se observó una disminución de los individuos del género Micrathena durante la época seca $\mathrm{y}$ un incremento de las poblaciones durante los meses con lluvias. Para el caso de $M$. schreibersi (Figura 4A), se observó que los individuos adultos (particularmente hembras) fueron más frecuentes durante dos períodos: el primero entre noviembre y diciembre y el segundo entre mayo y julio. Estos períodos correspondieron a los picos más altos de precipitación durante el estudio. Por otro lado, en relación a los individuos juveniles de esta especie, a diferencia de los adultos, sus mayores valores de abundancia fueron registrados a finales de la época seca (marzo) hasta principios de la segunda época de precipitaciones (mayo). Para las otras especies registradas, aunque presentaron un bajo número de individuos, observamos un patrón temporal similar al de $M$. schreibersi (Figura 4B y C). Además, la abundancia de las tres especies presentó una correlación positiva con la precipitación y la humedad, y una correlación negativa con la temperatura. Sin embargo, sólo M. schreibersi presentó una correlación significativa con la precipitación, mientras que $M$. quadriserrata, con la humedad (Tabla 2).

En cuanto a las tallas promedio, en el caso de las hembras se pudo apreciar que $M$. schreibersi fue la especie que presentó la mayor talla $(\mathrm{LO}=8.2 \mathrm{~mm}, \mathrm{AO}=5.7 \mathrm{~mm}$ ), seguida por M. sexspinosa $(\mathrm{LO}=5.4 \mathrm{~mm}, \mathrm{AO}=3.9 \mathrm{~mm}), M$.
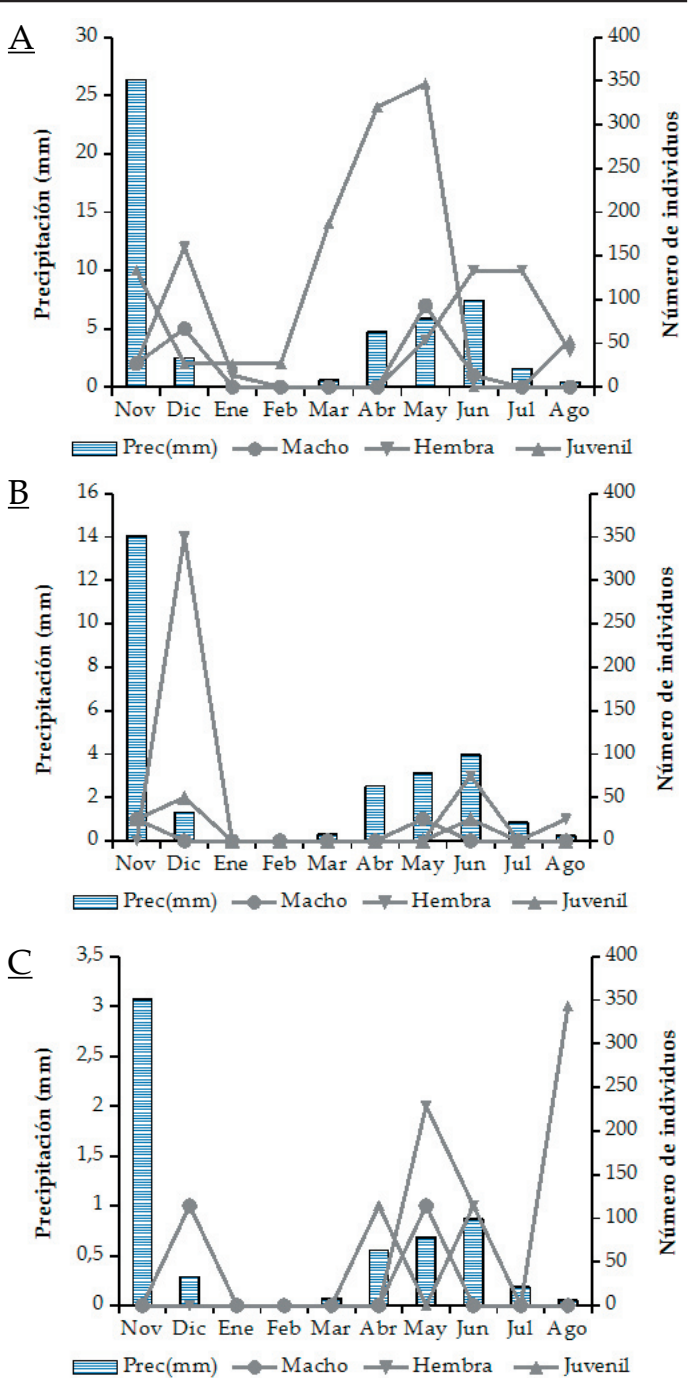

Figura 4. Variación mensual de las poblaciones de tres especies Micrathena en la RCM. A) M. schreibersi, B) $M$. sexspinosa y C) M. quadriserrata.

Figure 4. Monthly variation of three Micrathena species populations in the RCM. A: M. schreibersi, B) M. sexspinosa and C) M. quadriserrata. 
Tabla 2. Valores de correlación y probabilidad entre las abundancias de tres especies Micrathena y variables ambientales medidas durante el estudio en la RCM.

Table 2. Correlation and probability values between the abundances of three Micrathena species and environmental variables measured during the study in the RCM.

\begin{tabular}{lcccccc}
\hline & \multicolumn{2}{c}{ M. } & \multicolumn{2}{c}{$M}$. & \multicolumn{2}{c}{ M. } \\
Variables & \multicolumn{2}{c}{ schreibersi } & \multicolumn{2}{c}{ sexspinosa } & \multicolumn{2}{c}{ quadriserrata } \\
& $\mathrm{r}$ & $P$ & $\mathrm{r}$ & $P$ & $\mathrm{r}$ & $P$ \\
\hline Prec $(\mathrm{mm})$ & $0.71^{*}$ & 0.02 & $0.61^{*}$ & 0.06 & 0.29 & 0.41 \\
H.R. $(\%)$ & 0.43 & 0.21 & 0.38 & 0.28 & $0.77^{*}$ & 0.008 \\
T.A. $\left({ }^{\circ} \mathrm{C}\right)$ & -0.47 & 0.17 & -0.05 & 0.89 & -0.45 & 0.19 \\
\hline
\end{tabular}

quadriserrata $(\mathrm{LO}=4.2 \mathrm{~mm}, \mathrm{AO}=3.2 \mathrm{~mm})$ y $M$. horrida $(\mathrm{LO}=3.4 \mathrm{~mm}, \mathrm{AO}=4.0 \mathrm{~mm})$. En el caso de los machos, se invierte el patrón observado para las hembras debido a que $M$. quadriserrata $(\mathrm{LO}=3.6 \mathrm{~mm}, \mathrm{AO}=1.7 \mathrm{~mm})$ presentó los individuos de mayor talla, seguidos por los de $M$. schreibersi $(\mathrm{LO}=3.1 \mathrm{~mm}, \mathrm{AO}=1.2 \mathrm{~mm})$ y M. sexspinosa $(\mathrm{LO}=2.4 \mathrm{~mm}, \mathrm{AO}=0.9 \mathrm{~mm})$ (Figura 5).

\section{Discusión}

Las especies de Micrathena encontradas en este estudio presentan un patrón de variación temporal, caracterizado por la presencia de individuos adultos durante la época de lluvias, su desaparición durante la sequía y un aumento de juveniles al inicio de las lluvias. Este patrón es común al reportado para otras especies de arañas (Lubin 1978; Ferreira-Ojeda et al. 2009; Escorcia et al. 2012) y grupos de artrópodos (Jiménez-Sánchez et al. 2009; Barraza et al. 2010; Martínez et al. $2010 \mathrm{a}, \mathrm{b})$ presentes en bosques tropicales en donde la época seca genera un déficit hídrico que conlleva a cambios estructurales en la vegetación (Pizano et al. 2014) y, por lo tanto, un aumento en la temperatura del bosque, una disminución de la humedad relativa, de las presas potenciales y de los soportes para las telas. Estas variables fueron identificadas como limitantes para la permanencia de las arañas en ecosistemas naturales (Eberhard 1971; Uetz 1990).

La presencia de individuos adultos durante las épocas de mayor precipitación indicaría que durante este período se estarían llevando a cabo los eventos reproductivos, que se ven beneficiados por la mayor disponibilidad de alimento y de espacio (Didham and Springate 2003; Jiménez-Sánchez et al. 2009; Wolda 1978). Al finalizar la etapa reproductiva y realizar las puestas, la mayoría de las arañas orbitelares mueren (Foelix 2011), lo cual puede estar explicando la desaparición de las arañas adultas del genero Micrathena en la RCM durante la época seca.

Como mecanismo para asegurar la permanencia de la población en la próxima época de lluvias, las especies aquí estudiadas atraviesan el periodo climático extremo (seco en el BST) en forma de huevo. Este caso es parecido al descripto durante el invierno en México por Meling-López et al. (2008) como estrategia de sobrevivencia de M. funebris, y al reportado por Schaefer (1977) para algunas especies de las familias

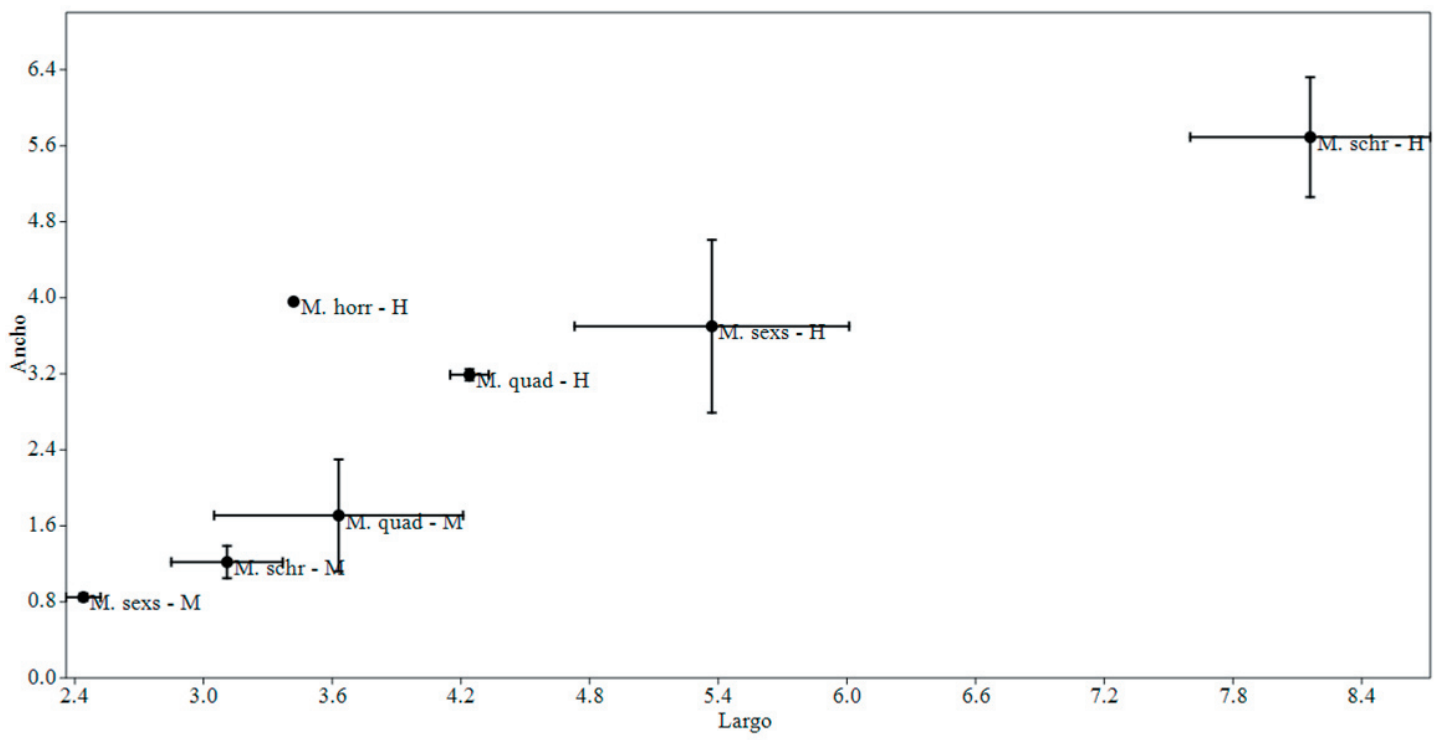

Figura 5. Promedios de talla de las Micrathena encontradas en la RCM. Las barras corresponden a la desviación estándar.

Figure 5. Average size of Micrathena found in the RCM. The bars correspond to the standard deviations. 
Araneidae y Linyphiidae en Alemania. La viabilidad de esta estrategia radica en las propiedades aislantes de la seda que recubre los ovisacos, que mantiene estable la humedad y la temperatura necesarias para el desarrollo normal de los huevos (Foelix 2011). En algunos artrópodos se ha probado experimentalmente que la eclosión de sus huevos está relacionada con el cambio en las condiciones ambientales (temperatura) (Hueck 1951; Cranham 1972; Parnrong and Cambell 2001; Zindler 2007). En este sentido, la llegada de las lluvias en el BST propicia la aparición de dichas condiciones (menor temperatura y mayor humedad), lo que favorecería la eclosión de los huevos en Micrathena. Esto explicaría la presencia de individuos inmaduros en la zona de estudio durante el inicio de las épocas de precipitación.

Las diferencias marcadas en proporción sexual encontradas en este estudio pueden ser atribuidas a tres factores: el tamaño reducido de los machos, los patrones sencillos de coloración y forma en comparación con las hembras y su alta movilidad en el hábitat en busca de pareja. Las anteriores variables pudieron disminuir la visualización y probabilidad de encuentros de individuos de este sexo, lo cual habría causado sus valores bajos de abundancia. Sin embargo, en arañas, los estudios relacionados con las proporciones sexuales en las poblaciones demostraron que las razones de tales desproporciones son variables y se relacionan con controles precigóticos estrictos y variaciones comportamentales y ambientales (e.g., la selección diferencial de esperma por las hembras durante la fecundación [Aviles and Maddison 1991; Hurst and Vollrath 1992], el desarrollo de individuos por partenogénesis [Camacho 1994], la posición del abdomen adoptada por las hembras durante y después de la copula [Gunnarsson et al. 2004] y el efecto de la mortalidad inducida por la severidad del ambiente y la presión de selección de depredadores [Gunnarsson 1987]). Estos estudios tuvieron gran auge durante algún tiempo en especies de hábitos sociales como Anelosimus eximius (Keyserling, 1884) (Aviles 1986; Vollrath 1986) y Australomisidia socialis (Main, 1988) (Rowel and Main 1992) y en la araña solitaria Pityohyphantes phrygianus (C. L. Koch, 1836) (Gunnarsson 1987; Gunnarsson 1989; Gunnarsson and Andersson 1992; Gunnarsson et al. 2004).

Por otro lado, los tamaños diferenciales de las especies de Micrathena de este estudio y su ocurrencia durante las mismas épocas del año, en parte sugiere que, para evitar la competencia por espacio y alimento, estas especies exhiben como posible mecanismo un patrón de segregación de nicho, relacionado con el consumo de presas con tamaños diferenciales. Esto ha sido previamente reportado para arañas que habitan en simpatría y que pertenecen al mismo gremio, como las especies aquí estudiadas. El tamaño de las arañas se ha reportado a menudo como una de las variables responsables de la coexistencia en el grupo (Uetz 1977), debido a que se relaciona con factores como el tamaño y el tipo de presa, así como con la dimensión de la tela. Esto implica que poseer cuerpos con tamaños diferenciales puede causar divergencia en el tipo de dieta que consumen las especies. Sin embargo, este es un tema controversial dentro del grupo (Wise 1995), y la coexistencia, en adición a lo aquí planteado, puede estar relacionada con factores como aislamiento en el plano vertical en la vegetación (Brown 1981) u horizontal en el ecosistema (Suwa 1986; Tretzel 1955), preferencias en el tipo de microhábitat (Greenstone 1980), en los patrones de actividad diaria (Wise 1995) o temporada de ocurrencia en el año (Uetz 1977), entre otras cuestiones relacionadas con la historia natural de las especies.

Los hallazgos de esta investigación demuestran que la biota presente en ecosistemas como el BST exhiben patrones ecológicos asociados con las condiciones estacionales del ecosistema. En el contexto actual de crisis de biodiversidad provocado por las actividades de origen antrópico, conocer las variaciones en las abundancias y su relación con variaciones climáticas es una excelente herramienta capaz de brindar información necesaria para crear planes de conservación y manejo de ecosistemas amenazados (e.g., el BST) (Pizano and García 2014). Las arañas del género Micrathena, debido a su relación con los factores ambientales y a la facilidad para identificarlas, verlas y capturarlas en el campo (Sabogal and Flórez 2000), se convierten en un buen grupo bioindicador. Por esta razón recomendamos su uso en futuros estudios de impacto ambiental.

Agradecimientos. A M. Cuervo por su colaboración económica para la realización de las jornadas de campo. A todas las personas de la Reserva Campesina La Montaña (RCM), por su apoyo en la fase de campo; en especial a Santiago "Chago" y a Rafael "El Mono". A la estudiante Z. Mejía por su colaboración en la determinación de los especímenes y al profesor 
E. Flórez por su ayuda en la confirmación de los ejemplares. Por último, agradecemos a la Facultad de Ciencias Básicas de la Universidad del Atlántico por facilitar el ingreso al laboratorio de Ecología y Entomología y a los dos revisores anónimos del manuscrito $\mathrm{y}$ al editor A. Farji-Brener, por su valiosa contribución para la mejora de este.

\section{REFERENCIAS}

Avilés, L. 1986. Sex-ratio bias and possible group selection in the social spider Anelosimus eximius. Am Nat 128:1-12.

Avilés, L., and W. Maddison. 1991. When is the sex ratio biased in social spiders? Chromosome studies of embryos and male meiosis in Anelosimus species (Araneae, Theridiidae). J Arachnol 19:126-135.

Barraza J., J. Montes, N. Martínez, and D. Cuauhtémoc. 2010. Ensamblaje de escarabajos coprófagos (Scarabaeidae: Scarabaeinae) del bosque tropical seco, Bahía Concha, Santa Marta (Colombia). Revista Colombiana de Entomología 36:285-291.

Barriga, J., and A. Moreno. 2013. Listado de las Arañas de Colombia (Arachnida: Araneae). Biota Colombiana 14(3): 21-33.

Beltrán, W., and J. M. Wunderle. 2014. Temporal Dynamics of Arthropods on Six Tree Species in Dry Woodlands on the Caribbean Island of Puerto Rico. J Insect Sci 14(199):1-11.

Berdugo-Latke, M. L., A. Avella, and J. O. Rangel-Ch. 2012. Patrón biotipológico foliar en un gradiente de precipitación en la Región Caribe de Colombia. Pp. 601-651 en J. O. Rangel-Ch (ed.). Colombia Diversidad Biótica XII: La región Caribe de Colombia. Instituto de Ciencias Naturales-Universidad Nacional de Colombia, Bogotá, Colombia.

Blanco, E., G. Amat, and E. Flórez. 2003. Araneofauna orbitelar (Araneae: Orbiculariae) de los Andes de Colombia: comunidades en hábitats bajo regeneración. Revista Ibérica de Aracnología 7:189-203.

Brown, K. M. 1981. Foraging ecology and niche partitioning in orb-weaving Spiders. Oecologia 50:380-385.

Bukowski, T., and T. Christenson. 1997. Natural history and copulatory behavior of the spiny orbweaving spider Micrathena gracilis (Araneae, Araneidae). J Arachnol 25:307-320.

Bukowski, T., and T. Christenson. 2000. Determinants of Mating Frequency in the Spiny Orbweaving Spider, Micrathena gracilis (Araneae: Araneidae). Journal of Insect Behavior 13(3):331-352.

Camacho, F. 1994. Female-biased sex ratio in spiders caused by parthenogenesis? Hereditas 120:183- 185.

Campuzano, E. F., G. Ibarra-Núñez, E. Chamé-Vázquez, and H. Montaño-Moreno. 2016. Understory spider assemblages from a cloud forest in Chiapas, Mexico, and their relationships to environmental variables. Arthropod-Plant Interactions 10(3):237-248.

Cranham, J. E. 1972. Influence of temperature on hatching of winter eggs of fruit-tree red spider mite, Panonychus ulmi (Koch). Ann Appl Biol 70:119-137.

Didham, R. K., and N. Springate. 2003. Determinants of temporal variation in community structure. Pp. 28-31 in Y. Basset, V. Novotny, S. E. Miller and R. L. Kitching (eds.). Arthropods of tropical forests: spatio-temporal dynamics and resource use in the canopy. Cambridge University Press. Cambridge, United Kingdom.

Eberhard, W. 1971. The ecology of the web of Uloborus diversus (Araneae: Uloboridae). Oecología 6:328-342.

Eberhard, W. 1988. Behavioral flexibility in orb web construction: effects of supplies in different silk glands and spider size and weight. J Arachnol 16:295-302.

Escorcia, R., N. Martínez, and J. Silva. 2012. Estudio de la diversidad de arañas de un Bosque Seco Tropical (Bs-T) en Sabanalarga, Atlántico, Colombia. Bol Cient Mus Hist Nat U de Caldas 16(1):247-260.

Ferreira-Ojeda, L., E. Flórez-Daza, and A. Sabogal-González. 2009. Arañas orbitelares de un bosque húmedo subtropical de la Sierra Nevada de Santa Marta (Magdalena, Colombia). Caldasia 31(2):381-391.

Foelix, R. 2011. Biology of spiders. 3rd ed. Oxford University Press, New York, USA.

Gálvez, D. 2011. Web decoration of Micrathena sexpinosa (Araneae: Araneidae): a frame-web-choice experiment with stingless bees. J Arachnol 39(1):128-132.

Garcia-Atencia, S., and N. Martínez-Hernández. 2015. Escarabajos fitófagos (Coleoptera: Scarabaeidae) del departamento del Atlántico, Colombia. Acta Zoológica Mexicana (nueva serie) 31(1):89-96.

Giraldo, A., D. Pérez, and G. Arellano. 2004. Respuesta de la comunidad de arañas epigeas (Araneae) en las lomas de Lachay, Perú, ante la ocurrencia del evento El Niño 1997-98. Ecología Aplicada 3(1,2):45-58.

Greenstone, M. H. 1980. Contiguous allotopy of Pardosa ramulosa and Pardosa tuoba (Araneae: Lycosidae) in the San Francisco Bay Region, and its implications for patterns of resource partitioning in the genus. American Midland Naturalist 104:305-311.

Grimbacher, P. S., and N. E. Stork. 2009. Seasonality of a diverse beetle assemblage inhabiting lowland tropical rain forest in Australia. Biotropica 41:328-337.

Gunnarsson, B. 1987. Sex ratio in the spider Pityohyphantes phrygianus affected by Winter severity. J Zool Lond 213: 609-619.

Gunnarsson, B. 1989. Local adjustment of sex ratio in the spider Pityohyphantes phrygianus. J Zool Lond 217:1-7.

Gunnarsson, B., and A. Andersson. 1992. Skewed primary sex ratio in the solitary spider Pityohyphantes phrygianus. Evolution 46:841-845.

Gunnarsson, B., G. Uhl, and K. Wallin. 2004. Variable Female Mating Positions and Offspring Sex Ratio in the Spider 
Pityohyphantes phrygianus (Araneae: Linyphiidae). Journal of Insect Behavior 17(1):129-144.

Hammer, Ø., D. Harper, and P. Ryan. 2001. PAST: Paleontological Statistics Software Package for Education and Data Analysis. Palaeo Electro 4(1):1-9.

Hodge, M. 1987a. Macrohabitat selection by the orb weaving spider, Micrathena gracilis. Psyche 94:347-361.

Hodge, M. 1987b. Factors influencing web site residence time of the orb weaving spider, Micrathena gracilis. Psyche 94:363-371.

Hueck, H. L. 1951. Influence of light upon the hatching of winter eggs of the fruit tree red spider mite. Nature 167: 993-994.

Hurst, L. D., and F. Vollrath. 1992. Sex-ratio adjustment in solitary and social spiders. Trends Ecol Evol 7:326-327.

Jiménez-Sánchez, E., S. Zaragoza-Caballero and F. A. Noguera. 2009. Variación temporal de la diversidad de estafilínidos (Coleoptera: Staphylinidae) nocturnos en un bosque tropical caducifolio de México. Revista Mexicana de Biodiversidad 80:157-168.

Levi, H. 1985. The spiny orb-weaver genera Micrathena and Chaetacis (Araneae: Araneidae). Boll Mus Compa Zoo 150(8):428-618.

Levi, H. 2002. Keys to the genera of araneid orbweavers (Araneae, Araneidae) of the Americas. J Arachnol 30:527562

Lubin, Y. D. 1978. Seasonal abundance and diversity of web-building spiders in relation to habitat structure on Barro Colorado Island, Panama. J Arachnol 6:31-51.

Magalhães, I. L. F and A. J. Santos. 2011. Two new species and taxonomic notes on the Neotropical spiny orb-weaving spiders Micrathena and Chaetacis (Araneae: Araneidae), with remarks on the development of Micrathena excavata. Zootaxa 2983:39-56.

Martínez N., L. Cañas, J. Rangel, O. Blanco, J. Mendoza, and S. Choen 2010a. Coleópteros coprófagos (Scarabaeidae, Scarabaeinae) en la reserva natural Las Delicias (RND), Sierra Nevada de Santa Marta (SNSM), Colombia. Bol Cient Mus Hist Nat U de Caldas 14:187-200.

Martínez, N., L. Cañas, J. Rangel, J. Barraza, J. Maarit, and O. Blanco. 2010b. Coleópteros coprófagos (Scarabaeidae: Scarabaeinae) en un fragmento de bosque seco tropical en el departamento del Atlántico, Colombia. Boletín del Museo de Entomología de la Universidad del Valle. 11:21-30.

MCcravy, K., and S. Hessler. 2012. Abundance and web characteristics of Micrathena gracilis and Micrathena mitrata (Araneae: Araneidae) in west-central Illinois, USA. J Arachnol 40(2):215-217.

McHugh, A., C. Yablonsky, G. Binford and I. Agnarsson. 2014. Molecular phylogenetics of Caribbean Micrathena (Araneae: Araneidae) suggests multiple colonization events and single island endemism. Invertebr Syst 28:337-349

Meling-López, A., C. Martínez-Camacho, and G. Duarte-Fuentes. 2008. Estructura poblacional y biología de la araña espinosa Micrathena funebris (Marx, 1898) del manglar del estero La Cruz, Bahía Kino, Sonora. BIOtecnia 10(3):3847.

Michalko, R., O. Kosulic, and V. Hula. 2016. Niche differentiation of two sibling Wolf spider species, Pardosa lugubris and Pardosa alacris, along a canopy openness gradient. J Arachnol 44(1):46-51.

Mineo, M. F., K. Del-Claro, and A. D. Brescovit. 2010. Seasonal variation of ground spiders in a Brazilian Savanna. Zoologia 27(3):353-362.

Parnrong, S., and I.C. Campbell. 2003. The effects of temperature on egg hatching of the mayfly Austrophlebioides marchanti (Ephemeroptera: Leptophlebiidae). Pp. 189-193 in E. Gaino (ed.). Research update on Ephemeroptera and Plecoptera. Università di Perugia. Perugia, Italy.

Pinheiro, F., I. R. Diniz, D. Coelho, and M. P. S. Bandeira. 2002. Seasonal pattern of insect abundance in the Brazilian Cerrado. Austral Ecology 27:132-136.

Pizano, C., R. González, M. F. González, F. Castro-Lima, R. López, N. Rodríguez, Á. Idárraga-Piedrahíta, W. Vargas, H. Vergara-Varela, A. Castaño-Naranjo, W. Devia, A. Rojas, H. Cuadros, and J. Lázaro. 2014. Las plantas de los bosques secos de Colombia. Pp. 48-93 en C. Pizano and H. García (eds.). El Bosque Seco Tropical en Colombia. Instituto de Investigación de Recursos Biológicos Alexander von Humboldt (IAvH). Bogotá, DC, Colombia.

Rangel-Acosta, J., O. Blanco-Rodríguez and N. Martínez-Hernández. 2016. Escarabajos copro-necrófagos (Scarabaeidae: Scarabaeinae) en diferentes usos del suelo en la Reserva Campesina la Montaña (RCM) en el departamento del Atlántico, Colombia. Bol Cient Mus Hist Nat U de Caldas 20 (1): 78-97. DOI : 10.17151/bccm.2016.20.1.7.

Rangel-Ch, J. O., and J. E. Carvajal-Cogollo. 2012. Clima de la Región Caribe Colombiana. Pp. 67-129 en Rangel-Ch, J. O. (ed.). Colombia Diversidad Biótica XII: La Región Caribe de Colombia. Instituto de Ciencias Naturales-Universidad Nacional de Colombia, Bogotá, Colombia.

Richardson, M. L., and L. M. Hank. 2009. Partitioning of Niches Among Four Species of Orb-Weaving Spiders in a Grassland Habitat. Environ Entomol 38(3):651-656.

Sabogal, A., and E. Flórez. 2000. Arañas espinosas del género Micrathena Sundevall, 1833 (Araneae: Araneidae) de Colombia. Biota Colombiana 1(3):253-260.

Schaefer, M. 1977. Winter ecology of spiders (Araneida). Z Ang Ent 83:113-134.

Shelly, T. 1984. Prey selection by the neotropical spider Micrathena schreibersi with notes on web-site tenacity. Proc Entomol Soc Wash 86:493-502.

Sudhikumar, A. V., M. J. Mathew, E. Sunish, and P. A. Sebastian. 2005. Seasonal variation in spider abundance in Kuttanad rice agroecosystem, Kerala, India (Araneae). Acta Zool Bulg 1:181-190. 
Suwa, M. 1986. Space partitioning among the wolf spider Pardosa amentata species group in Hokkaido, Japan. Researches on Population Ecology 28:231-252.

Tretzel, E. 1955. Intragenerische Isolation und interspezifische Konkurrenz bei Spinnen. Zeitschrift fur Morphologie und Okologie. Tierre 44:43-162.

Uetz, G. 1990. Prey selection in web-building spiders and evolution of prey defenses. Pp. 93-128 in D. L. Evans and J. O. Schmidt (eds.). Adaptative mecanisms and strategies of prey and predators. SUNY Press, New York, EU.

Uetz, G., and S. Hartsock. 1986. Prey selection in an orb-weaving spider: Micrathena gracilis (Araneae: Araneidae). Psyche 94:103-116.

Uetz, G. W. 1977. Coexistence in a guild of wandering spiders. Journal of Animal Ecology 46:531-542.

Vanderhoff, E., C. Byers, and C. Hanna. 2008. Do the Color and Pattern of Micrathena gracilis (Araneae: Araneidae) Attract Prey? Examination of the Prey Attraction Hypothesis and Crypsis. J Insect Behav 21:469-475.

Vasconcellos, A., R. Andreazze, A. M. Almeida, H. F. P. Araujo, E. S. Oliveira, and U. Oliveira. 2010. Seasonality of insects in the semi-arid Catinga of northeastern Brazil. Rev Bras Entomol 54:471-476.

Vollrath, F. 1986. Eusociality and extraordinary sex ratios in the spider Anelosimus eximius (Araneae: Theridiidae). Behav Ecol Sociobiol 18:283-287.

Ward, D., and Y. Lubin. 1992. Temporal and spatial segregation of web-buildin $\mathrm{g}$ in a community of orb-weaving spiders. J Arachnol 20:73-87.

Wise, D. H. 1993. Spiders in Ecological Webs. Cambridge University Press. New York, United State.

Wolda, H. 1978. Seasonal fluctuations in rainfall, food and abundance of tropical insects. J Anim Ecol 47:369-381.

Wolda, H. 1988. Insect seasonality: why? Annu Rev Ecol Syst 19:1-18.

World Spider Catalog. 2017. World Spider Catalog. Natural History Museum Bern, online at http://wsc.nmbe.ch, version 18.0 , accessed on 23/03/2017.

Zindler, C. 2007. The effects of temperature and humidity on the eggs of Aedes aegypti (L.) and Aedes albopictus (Skuse) in Texas. PhD. Texas A\&M University. Texas, EU. 